\section{A COMPACT PORTABLE HF TERMINAL}

M Damell, B Honary, R Enright and I Martin

HW Communications Ltd, UK

\begin{abstract}
The paper describes the design philosophy and implementation of a compact, highperformance $\mathrm{HF}(2-30 \mathrm{MHz})$ long-range radio system. The system is automatic and can adapt to changes in the propagation and cochannel interference environment. In the design, extensive use is made of advanced real-time DSP algorithms and technology; this allows relatively simple RF equipment to be exploited, which leads to an economic system implementation. The system is readily transportable in a suitcase-sized container.
\end{abstract}

\section{INTRODUCTION: APPLICATION AREAS}

In the HF (2 - $30 \mathrm{MHz}$ ) band, propagation via both surface wave and ionospheric refraction mechanisms allows point-to-point communication over distances from line-ofsight (LOS) to many thousands of miles [1] The highly variable nature of the ionospheric propagation medium makes it difficult to use consistently and efficiently. High reliability can however be achieved through the use of transmitter powers of several kilowatts and large directional antennas; hence, the installation and support costs are high. The amount of information which can be passed over an HF channel is restricted by the available channel bandwidth of typically $3 \mathrm{kHz}$. Digital data rates of up to a few hundreds of bits/second or analogue speech are the most common traffic formats [2].

The major impetus for the development of HFtype radio communication systems has in the past been provided by defence requirements, particularly where mobile terminals such as ships and aircraft are involved. Over the past two or three decades, there has been a move away from HF systems as a primary form of long-range, beyond LOS (BLOS) communication and towards the use of satellite facilities [3]. As a consequence, R\&D funding for improving HF-type systems has tended to diminish. There are still some residual operational requirements which can only be met by HF systems; however, the cost of equipment to meet these requirements is relatively high.

The rationale for the civil use of HF radio links to provide long-range communications is largely cost-dependent; if they can be implemented at low cost, with an acceptable level of reliability and traffic throughput, they become attractive to potential users since they provide the basis of a fully-autonomous communications system. This is in contrast to satellite systems where users are totally dependent upon the access facilities provided by a third party. HF terminals can also be simply transported to different locations, if required. At present, the high cost of HF equipment (largely to military specifications) makes it uncompetitive in many civil situations where, technically, it is well able to fulfil the user requirements.

Amateur radio enthusiasts make extensive use of the HF medium. Modern amateur-grade transceivers are reliable and have a good performance specification in some respects; in other respects, e.g. frequency stability, their performance is marginal for digital traffic.

It is the purpose of this paper to describe a compact and fully automatic HF radio system, capable of adaptive operation in response to path conditions. The cost of the system is much closer to that of amateur systems than professional/military-type systems, but its performance is comparable with that of the professional systems. The complete radio system terminal is contained in a volume equivalent to that of a medium-size suitcase and can be readily transported and operated in any part of the world. Thus, the system is potentially suited to a wide range of both military and civil requirements 


\section{GENERAL TECHNICAL APPROACH}

An overall schematic diagram of the system is shown in Figure 1. The system incorporates novel digital signal processing (DSP) algorithms for modulation/demodulation [4], frequency-offset correction [4], error control [5][6], synchronisation [7][8], real-time channel evaluation (RTCE) [9] and system control and adaptation [10].

The system design coalesces a range of techniques for reliable digital data transmission, all of which have been extensively validated via experimental trials, within the architecture of an advanced radio system. The system normally operates using a simplex/ARQ transmission protocol; however, half-duplex operation is also possible. Data messages can be input via a keyboard or other means and output via a VDU or other userspecified equipment; an analogue speech transmission mode is also available. The major elements of the proposed system at each terminal are:

\section{(a) an amateur-grade transceiver} ( maximum 100W output power);

(b) a sloping- $V$, or other simply depoyable, antenna for both transmission and reception;

(c) a 486-based laptop PC for overall system control and protocol generation;

(d) a PC DSP expansion card to perform realtime signal generation and processing functions (DSP-32C processor);

(e) flexible power supply facilities.

The general design philosophy is to compensate for lower transmitter powers, simple antennas and a degree of frequency instability in the transceiver by sophisticated signal processing and system control procedures. Thus, reduced dependence on expensive professional RF technology is compensated for by exploiting sophistication in the use of relatively cheap DSP technology to achieve the required system integrity and reliability. Also, the signal generation and processing algorithms are uniquely digital, rather than being simply digital equivalents of analogue functions.

Single sideband (SSB) operation enables all signal generation and processing functions to be carried out in a $3 \mathrm{kHz}$ baseband, thus allowing implementational simplicity and flexibility. Simple interfaces with the transceiver facilitate rapid system control and adaptation.

\section{FUNCTIONAL ELEMENTS OF THE SYSTEM}

The major functional elements of the suitcase HF system will now be described individually.

\subsection{Modem}

The suitcase HF system uses an MFSK modem, based on the PICCOLO concept [4], implemented via real-time DSP. The modem incorporates a novel intrinsic synchronisation scheme known as matched filter derived synchronisation, in which maximum likelihood symbol synchronisation is achieved by appropriate processing of the tone quadrature matched filter outputs; therefore, no specific synchronisation overheads are required. A variable synchroniser memory allows the effects of fading to be overcome. Initial block synchronisation is obtained by the use of a suitable preamble sequence [7].

Frequency offset or Doppler correction is carried out automatically to allow operation with non-professional RF equipment and over unstable propagation paths.

Tone frequencies are independently variable to an accuracy of $1 \mathrm{~Hz}$ over the transmission channel bandwidth; orthogonal or other tone formats can be selected. Also, tone positions can be adjusted to avoid sources of narrowband co-channel interference.

In summary, therefore, the modem parameters which can be adapted in response to path or equipment state are:

(a) number of tones: from 2 to 32 ;

(b) tone frequencies: all independently variable over nominal $3 \mathrm{kHz}$ bandwidth, in either orthogonal or non-orthogonal formats;

(c) synchroniser memory: up to 50 symbol intervals;

(d) number of samples per symbol interval: up to 30 ; 
(e) symbol rate: up to $500 / \mathrm{s}$ with limited tone set;

(f) frequency offset correction: up to $+/-140 \mathrm{~Hz}$;

(g) preamble format: binary or multilevel with different lengths.

\subsection{Codec}

The error control codec for the system is based on the use of Reed-Solomon (RS) codes [11]. These codes are decoded using the minimum weight algorithm [12], with the codec being implemented via real-time DSP. The minimum weight decoder (MWD) can be configured to perform forward error correction (FEC) only in a duplex transmission mode, or to employ a hybrid FEC/error detection scheme in an automatic repeat request (ARQ) protocol.

The codec allows a code to be selected from a predetermined "library" of codes; the code set in use at any time is selected on the basis of channel state (RTCE) data, user requirements and operational protocol. The decoder itself also provides an RTCE metric data output. The following code parameters can be modified in response to variations in channel state:

(a) symbol alphabet;

(b) block length;

(c) information symbols/block;

(d)balance between error correction and detection.

\subsection{Real-time Channel Evaluation (RTCE)}

The ready availability of RTCE data is essential to the efficient and adaptive operation of the system. This is potentially available from a number of sources, i.e. modem, codec, passive monitoring, active channel probing, etc. [9]. In this context, it has been decided to use embedded RTCE metrics derived from the modem and codec. The fact that the system operates at present in low-speed data modes means that detailed ionospheric mode structure has relatively little importance since symbol intervals are generally long compared with multipath spread. Thus, metrics derived directly from information within the modem and codec, which can be simply calibrated in terms of signal-to-noise ratio (SNR), are employed.

\subsection{Control and Adaptation}

The system operates with either fixed or adaptive control protocols. As stated previously, it can operate in a balanced bidirectional mode or a predominantly unidirectional (ARQ) mode. Adaptation is in response to SNR estimates derived from modem/codec metrics.

The user interface is via a conventional laptop computer display and keyboard. Alternatively, data input to the system via a direct digital interface can be transmitted after storage and output from a suitable buffer; output rates from the buffer are determined by channel state.

\subsection{Power Supplies}

Flexible power supplies are provided to allow the system to operate from a wide range of DC and $A C$ sources.

\section{CONCLUDING REMARKS}

This paper describes the principles of operation and practical configuration of an advanced, portable and economic HF radio system, designed for simple deployment in a wide range of operational situations. The system currently operates in low-speed data transmission modes; it can, however, be extended to transmit analogue speech, medium-rate data and digitised image information. 


\section{REFERENCES}

[1] Davies, K.: "Ionospheric radio", Peter Perigrinus, 1990.

[2] Darnell, M.: "HF system design", NATOAGARD Lecture Series No. LS-145 on "Propagation impact on modern HF communication system design", Belgium/France/Denmark, April 1986.

[3] Maslin, N.: "HF communications: a systems approach", Pitman, 1987.

[4] Clark, P.D.J., Horley, N., Darnell, M. and Honary, B.: "A DSP-based MFSK modem", IEEE Conf. MILCOM '92 (USA), Oct. 1992.

[5] Darnell. M., Honary, B. and Zolghadr, F.: "Embedded coding technique: principles and theoretical studies", Proc. IEE-F, Vol. 135, No. 1,1988 .

[6] Honary, B., Arani. F.S. and Darnell, M.: "Embedded modulation and coding for HF channels", in "Cryptography and coding II", Oxford Univ. Press, 1992.

[7] Grayson, M. and Darnell, M.: "A DSP realisation of a multi-functional coding approach to word synchronisation", Int. Conf. on "DSP applications and technology", Berlin, Oct. 1991.

[8] Honary, B., Zolghadr, F. and Darnell, M.: "Code-assisted bit synchronisation (CABS) scheme", IEE Electronics Letters, Vol. 25, No. 2, Jan. 1989.

[9] Darnell, M.: "Embedded real-time channel evaluation techniques", as for [2].

[10] Darnell, M.: "Real-time channel evaluation techniques", Proc. 1st Int Symp. on "Communication theory and application", Paper No. 64, Crieff, Scotland, Sept. 1991.

[11] Reed, I.S. and Solomon, G.: "Polynomial codes over certain finite fields", J. Soc. Indust. \& Appl. Maths., Vol. 8, June 1960.

[12] Rice, M., Taleb, F. and Farrell, P.G.: "Minimum weight decoding", Tech. Note TN 1001, MAN Issue 1, Manchester Univ., May 1986. 


\begin{tabular}{|c|}
\hline $\begin{array}{c}\text { Portable H.F Terminal } \\
\text { Schematic Diagram }\end{array}$ \\
\hline
\end{tabular}

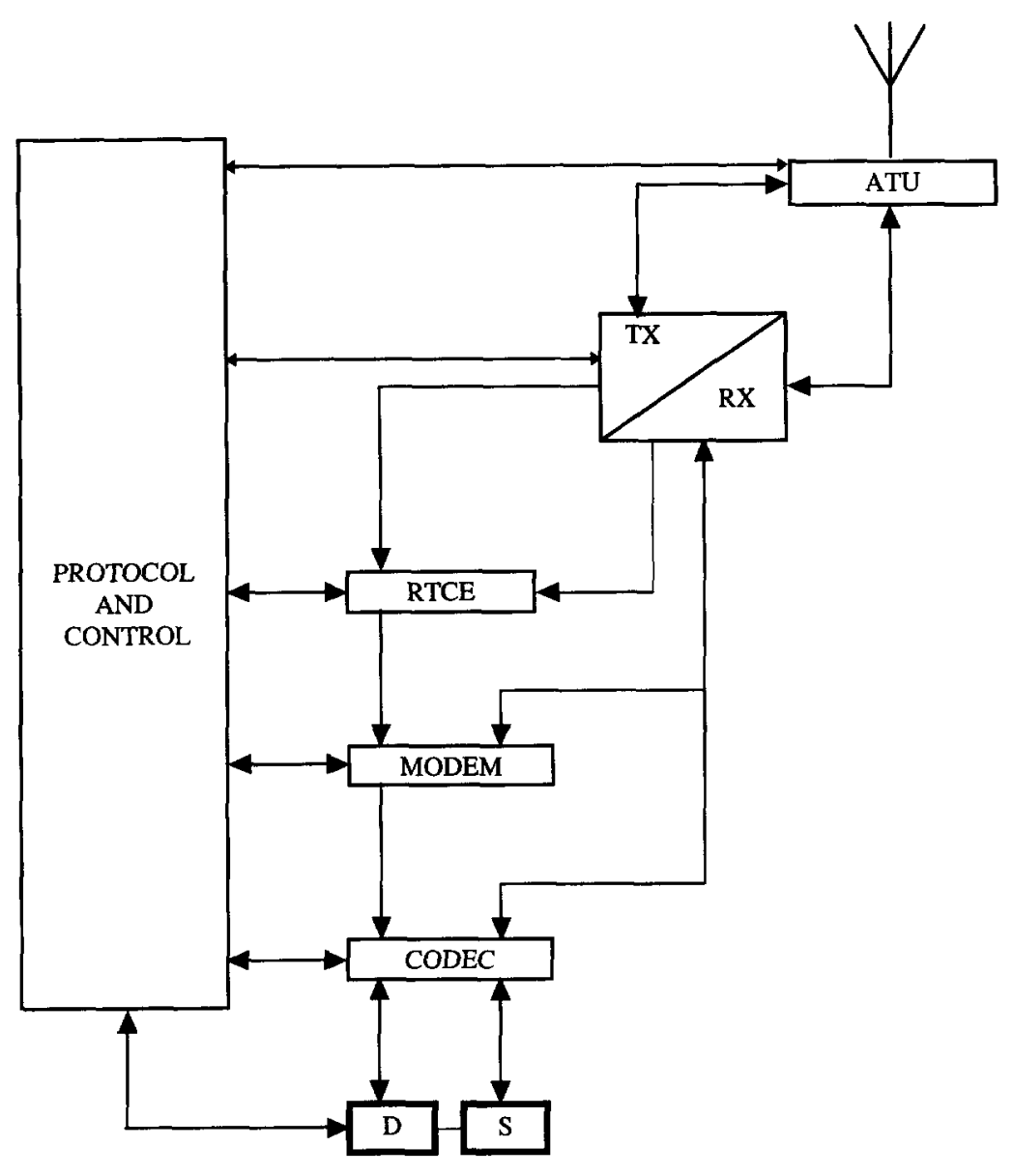

\section{User Interface}

D - Destination

S - Source

fig 1: Portable H.F. Terminal Schematic Diagram 